\title{
High voltage energy efficiency power supply
}

\author{
Sergei Makov*, Boris Kudryavcev, Ilya Grinev and Alexander Minaev \\ Institute of Services Sector and Entrepreneurship, Shakhty Branch of DSTU, Radio \\ Engendering and Electrotechnical Systems and Complexes Department, Russia
}

\begin{abstract}
This article discusses the selection of operating modes for the high voltage power supply of the electrostatic dust collector. Methods of high voltage generation, voltage regulation in converters and the choice of the converter operation mode are considered.
\end{abstract}

\section{Introduction}

During the past twenty years considerable progress has been made in the field of dust collection. The cyclone separator, one of the most common dust collectors, has been widely used in industrial production due to the advantages such as simple structure, easy operation, and low cost of operation and maintenance [1]. The dust particles can be separated from the airflow and trapped on the wall by the centrifugal force and then fall into the dust hopper. Larger-sized particles can be separated by this approach [2]. The main disadvantage of cyclone systems is the separation capacity has weaker efficiency for dust with smaller particle size.

Precipitators with electrostatic dust collection methods are used for dust of smaller particle size. Electrostatic precipitator (ESP) uses the electrostatic attraction to separate particulates present in suspension within a gaseous flow. In detail, these solid or liquid particles are positively or negatively charged thanks to the application of a high voltage electric field (corona discharge) [3]. Following this process, the electrically charged particles are deposited on the collecting electrode.

The first step in developing of a high-voltage dust collector power supply is to choose an approach to high voltage energy efficiency generation, choose a method of voltage regulation in converters and also choose an operating mode. In terms of energy efficiency the main problem is energy loss due to leakage on insulators and energy efficiency of the high voltage converter itself. These issues will be discussed below.

\section{Approaches to generation of high voltage}

This section will provide an overview of the existing approaches to the generation of high voltage. Higher energy efficiency can be achieved in two approaches: using smart control of voltage source and reducing loss in voltage source itself by choosing converter architecture.

*Corresponding author: makovs@ rambler.ru 


\subsection{Smart control}

The functional diagram of the smart control approach is shown in the figure 1 .

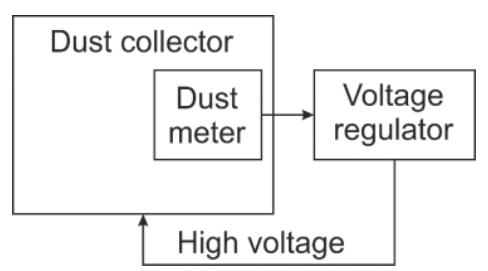

Fig. 1. Functional diagram of smart control

The dust-meter measures current dust level and sends the data to the voltage regulator. The voltage regulator changes the voltage level of the dust collector power supply depending on the received dust level data. The main idea of smart control is to change voltage or connect additional cleaning sections depending on the dust level. Dust analysis and control of the power supply increases the energy efficiency of the system because high voltage is used only when it is necessary.

\subsection{Voltage regulator architecture}

One of the most common approaches to high voltage generation is to use a transformer [4]. The output voltage of the transformer at harmonic impact can be calculated by the formula

$$
U_{\text {out }}=n U_{\text {in }}
$$

where $U_{\text {in }}$ - effective voltage at the input of the transformer, $n$ - transformation ratio.

This method for generating a high voltage does not allow the output voltage to be controlled and the output current is not limited. An alternating of pulsing voltage can be increased and simultaneously rectified using a cascade generator (also known as voltage multiplier). A voltage multiplier operation is based on rectifying overcharge current of capacitors [5]. The circuit of a half-wave three-cascade voltage multiplier is shown in fig. 2.

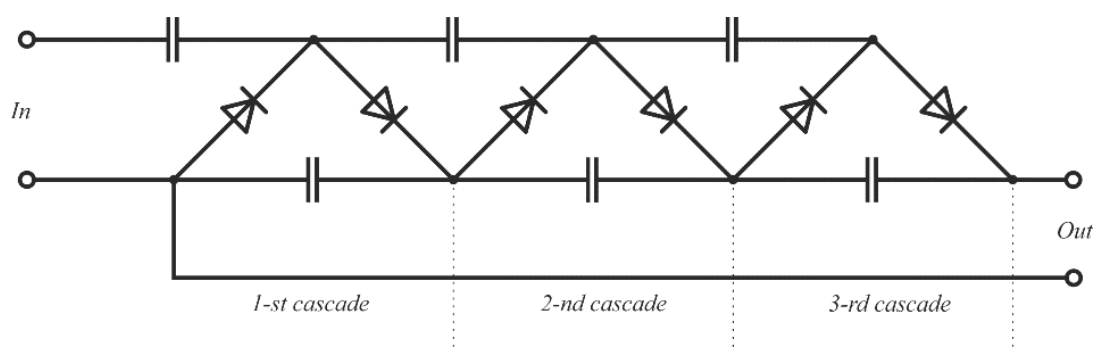

Fig. 2. Half-wave three-cascade voltage multiplier

The constant voltage at output of three-cascade half-wave voltage multiplier with a harmonic voltage at input can be calculated using formula: 


$$
U_{\text {out }}=2 n U_{\text {in }}-\frac{I_{H}}{\omega C} \cdot\left(\frac{2}{3} n^{3}+\frac{1}{4} n^{2}-\frac{1}{6} n\right)
$$

where $n$ - number of generator cascades;

$U_{i n}$ - the amplitude of the harmonic voltage at the input of the circuit;

$I_{H}$ - load current;

$\omega$ - circular frequency of harmonic voltage at input;

$C$ - capacitance of capacitor (same for all cascades);

From (2) we can see that output voltage strongly depends on current in load at a low frequency or low capacitance of capacitors. In practice, voltage at the output is also affected by dielectric losses in capacitors, which makes the use of more than 3 cascades impractical. This is the main drawback in usage of voltage multipliers [6].

To avoid disadvantages of transformer and voltage multiplier a diode-cascade transformer [7] is used. It consists of a voltage multiplier and transformer (fig. 3). This approach allows to use diodes with lower reverse voltage, and the output voltage can be calculated using the formula:

$$
U_{\text {out }}=n U_{\text {in }}-m U_{d f}
$$

where $n$ - ratio of number of turns in secondary windings to number of turns in primary winding;

$U_{\text {in }}$ - amplitude of the harmonic voltage at the input of the circuit;

$m$-number of cascade (diodes connected in series);

$U_{d f}$ - voltage drop through the diode when current flows in the "forward" direction.

Voltage at output of diode-cascade transformer does not depend on current in load, if active resistance of windings and losses in magnetic circuit are not taken into account [8].

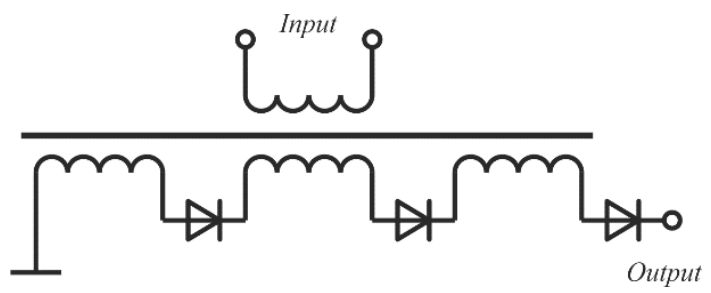

Fig. 3. Diode-cascade transformer

\subsection{Convertor architecture}

The use of a transformer to increase the voltage without additional circuit solutions does not allow regulation of the output voltage. Pulse-width modulation is used to regulate the voltage in pulse converters.

There are two modes for using a transformer when applying pulse width modulation [9]:

- forward converter (fig. 4);

- flyback converter (fig. 5).

The forward converter is a $\mathrm{DC} / \mathrm{DC}$ converter that uses a transformer to increase or decrease the output voltage (depending on the transformer ratio) and provide galvanic isolation for the load. With multiple output windings, it is possible to provide both higher and lower voltage outputs simultaneously. Primary winding current and key current directly 
depend on the current in the load in this mode. In this case, in a short circuit in a load, it is necessary to take measures to protect the key from overcurrent.

The flyback converter is an isolated power converter. The two prevailing control schemes are voltage mode control and current mode control (in the majority of cases current mode control needs to be dominant for stability during operation). Both require a signal related to the output voltage. There are three common ways to generate this voltage. The first is to use an optocoupler on the secondary circuitry to send a signal to the controller. The second is to wind a separate winding on the coil and rely on the cross regulation of the design. The third consists of sampling the voltage amplitude on the primary side, during the discharge, referenced to the standing primary DC voltage.

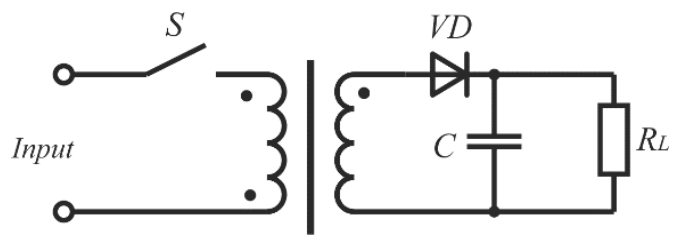

Fig. 4. Forward converter

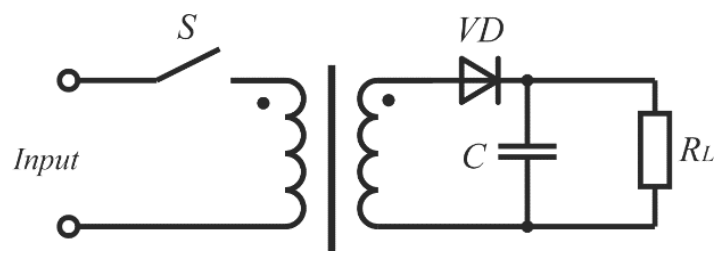

Fig. 5. Flyback converter

The advantage of a flyback converter is that there is no need to protect the key from overcurrent in case of a short circuit in load, since current of primary winding is determined only by its inductance and the duration of open state of the key. Flyback converter was chosen for use in the developed power supply for the dust collector because it is easier to provide short-circuit reliability in load.

The two common switching modes of operation of flyback are fixed frequency (FF) and quasi resonant (QR) [9]. The choice of switching mode depends on many factors such as power, efficiency, form factor, development time and so on. In our case, quasi resonant mode was chosen because it is more energy effective [10].

In comparison with both discontinuous mode and continuous mode operation of a flyback converter, quasi-resonant switching offers reduced turn on losses, resulting in increased efficiency and lower device temperature. The disadvantage of higher losses at light loads for a simple quasi-resonant circuit is removed by the frequency clamp circuit used in modern controllers or integrated power switches.

\section{Discussion}

Experimental modeling was carried out to confirm correct choice of approaches. Experimental modeling showed that the use of transformer architecture with cascade rectifier 
and flyback converter approach gave from $3 \%$ to $5 \%$ improvement in efficiency over forward converter. This result is explained by the reduction of losses on the transient of the converter key.

Modeling results are shown in table 1.

Table 1. Power loss.

\begin{tabular}{|c|c|c|c|}
\hline Mode & No load & Middle load & High load \\
\hline FF & $10 \mathrm{~W}$ & $23 \mathrm{~W}$ & $50 \mathrm{~W}$ \\
\hline QR & $7 \mathrm{~W}$ & $9 \mathrm{~W}$ & $10 \mathrm{~W}$ \\
\hline
\end{tabular}

According to results in table 1, we can see power loss is decreasing up to 5 times with using QR mode instead of FF mode.

\section{Conclusion}

In this article the selection of operating modes for the high voltage power supply of the electrostatic dust collector was discussed. The results of experimental modeling showed $3 \%$ to $5 \%$ increase in efficiency of transformer architecture with cascade rectifier and flyback converter approach over forward converter.

Additionally, we evaluate an additional from 3\% to $5 \%$ increase in efficiency due to the intelligent regulation of voltage at source depending on dust level.

\section{References}

1. Xie B. et al. Powder technology. 339. 695-701 (2018).

2. A.N. Huang, K. Ito, T. Fukasawa, H. Yoshida, H.P. Kuo, K. Fukui, Classification performance analysis of a novel cyclone with a slit on the conical part by CFD simulation, Sep Purif Technol, 190. 25-32 (2018).

3. Viegas C. et al. Air Quality, Atmosphere \& Health. 12.5. 573-583 (2019).

4. Naidu M. S. High voltage engineering. - Tata McGraw-Hill Education (2013).

5. Brugler J. S. IEEE Journal of Solid-State Circuits. 6. 3, 132-135 (1971).

6. Iqbal S. IEEE transactions on power electronics. 29. 1. 6-12 (2013).

7. Dash A. R. et al.International Transactions on Electrical Energy Systems. 29. 2. e2728 (2019).

8. Banaei M. R. et al. Journal of Electrical Engineering and Technology. 7. 4. 538-545 (2012).

9. Xie, Xiaogao, et al. Study on the single-stage forward-flyback PFC converter with QR control. IEEE Transactions on Power Electronics 31.1. 430-442 (2015).

10. Wang, Yijie, et al. Single-stage QR AC-DC converter based on buck-boost and flyback circuits. IET Power Electronics 10.1. 103-111 (2017). 